\title{
IV. DIE LETZTE CHANCE DAS FEST VOM 17. AUGUST 1661
}

Der Bau von Vaux-le-Vicomte und die damit verbundene Kulturpatronage sollten die Beziehung zwischen dem König und seinem Finanzminister stärken und Fouquet als loyalen Diener der Krone ins rechte Licht setzen, um ihm den Weg zum Amt des Premierministers zu ebnen. Doch gerade diese Abhängigkeit von einem Ersten Minister war es, die Ludwig XIV. hinter sich lassen wollte, als er nur einen Tag nach Mazarins Tod seine Alleinregierung erklärte. Der zwar 1654 gekrönte, doch bislang unter Mazarin wie unmündig gehaltene Ludwig übernahm selbst die Regierungsgeschäfte und erhob sich damit ein zweites Mal auf den Thron. Dadurch erfuhr auch Fouquets politische Lage eine Wende. Die visuelle Botschaft von Vaux-le-Vicomte wurde nicht nur hinfällig - sie verkehrte sich sogar in ihr Gegenteil. War es zu Zeiten Mazarins und seiner Regierung durchaus vertretbar gewesen, dem jungen und unerfahrenen König gegenüber die eigenen Führungsqualitäten zu rühmen, so bedeutete dies nach der Regierungsübernahme eines sich für mündig erklärenden Monarchen einen Affront. Die Wende von 1661 machte die baulichen Investitionen des Finanzministers zu einer Waffe, die sich gegen ihn selbst zu richten drohte.

Die Drehung des politischen Windes bekam Fouquet bereits wenige Tage nach der Regierungsübernahme durch Ludwig zu spüren: Der König forderte von dem Minister Rechenschaft über sein Finanzgebaren der letzten Jahre. Allein Fouquet sollte die Verantwortung für die Misere des Staatshaushalts tragen, obgleich er derjenige war, der die bereits $\mathrm{zu}$ seinem Amtsantritt schlechte Finanzlage der Krone über das Ende des Spanischen Krieges hinweg und trotz der unaufhörlichen pekuniären Forderungen Mazarins gerettet hatte. Fouquet versuchte alles, sich dem jungen Monarchen gegenüber als loyaler Diener zu beweisen. Er nahm alle Schuld für die leeren Staatskassen auf sich und bat den König offiziell um Verzeihung. Diese wurde ihm gewährt. Zugleich stellte man ihm keinen anderen als seinen Rivalen Colbert an die Seite, der fortan den Finanzminister kontrollieren sollte. Offiziell bestätigte man diesen in seinem Amt. In Wirklichkeit aber hatte Fouquet keine Chance mehr: Nur zwei Monate nach dem Tod Mazarins war sein Sturz beschlossene Sache. Der Finanzminister aber hoffte noch immer auf seine Rehabilitierung. Mit allen Mitteln versuchte er, den König von seinen Qualitäten zu überzeugen und verkaufte sogar sein Amt als Generalstaatsanwalt des Pariser Parlaments und die damit verbundene Rechtssicherheit, als Ludwig ihn um eine hohe Geldsumme bat. Auch seine Kulturpatronage richtete er fortan nicht mehr indirekt (wie zu Zeiten Vaux-le-Vicomtes), sondern direkt auf den Monarchen aus. Die Gemäldekäufe des Jahres 1661 durch Fouquet waren allesamt für die Vergrößerung der königlichen Sammlung gedacht. Der nach 
Rom entsandte Maucroix erhielt den Auftrag, nach Präsenten für den König und seine Familie Ausschau zu halten. Um auch sein zur Erlangung der königlichen Gunst erbautes Schloss Vaux-le-Vicomte zu rehabilitieren, nahm er die Bitte des Königs nach einem dortigen Besuch wahr, um ihm die Anlage in einer neuen Weise zu zeigen. Mit einem großartigen Einweihungsfest präsentierte der Finanzminister sich und seinen noch immer unvollendeten Bau am 17. August 1661 dem Monarchen.

Die höfische Festkultur hatte in Frankreich eine lange Tradition. Sowohl der königliche Hof selbst als auch die Schlösser und Stadthäuser der Aristokratie und der haute bourgeoisie waren Schauplätze festlicher Vergnügungen, die das Leben der höfischen Gesellschaft prägten:

Les Masques, durant la semaine,

Ont bien couru la pretentaine;

Et quelques Gaillards m'ont apris

Qu'en cette Ville de Paris,

On a donné neuf cens Aubades,

Dansé trois mille Mascarades,

Et, tant sérieux, que folets,

Deux cens cinquante et six Balets ${ }^{1}$.

Anfang des 17. Jahrhunderts gehörten noch Maskeraden, Waffenspiele und Turniere zum Kanon eines herrschaftlichen Festes. Im Laufe des Grand Siècle wurden die Inszenierungen pompöser und die Ausgestaltung durch namhafte Künstler gewann an Bedeutung. Die Begeisterung für aufwendige Ballettchoreographien und Theaterinszenierungen, prächtige Kostüme sowie innovative Maschinenkonstruktionen und pyrotechnische Vorführungen prägte nun die höfischen Feste. In der Mitte des 17. Jahrhunderts war es besonders unter den hohen Staatsbeamten zur Regel geworden, bei den Empfängen des Königs in ihren Schlössern ein reichhaltiges Bankett mit der Aufführung einer Komödie, eines Balletts oder einem Feuerwerk zu kombinieren. Über den Rahmen einer zweiphasigen Abendveranstaltung gingen die Empfänge für den König jedoch meist nicht hinaus. Nur selten findet sich in den Journalen der Zeit der »Gazette de France« oder der »Muze historique«, in denen die Empfänge überliefert sind - der Bericht eines Abends, bei dem alle Festelemente kombiniert waren. Ein solches Fest war der Empfang des Königs am 8. August 1651 durch den président des Comptes Tubeuf in seinem Schloss Rueil, bei dem nach einer Jagdpartie ein Ballett dargeboten wurde, auf welches die Aufführung einer Komödie folgte. Ein Imbiss und ein Feuerwerk schlossen das Fest $\mathrm{ab}^{2}$. Auf eine ähnliche Weise wurde der König am 18. Mai 1659 vom

${ }^{1}$ Muze historique, Bd. II, Lettre IX, 2. März 1658, S. 451. Einen Monat später heißt es in der »Muze historique «: »Il est vray que pour un repas dressé par ordre et par compas, et pour sçavoir servir des tables de mets friands et délectables, avéques somptüozité, dans Paris, n'est pas rareté; faire un Festin copieux, ample, n'est pas un acte sans exemple « (ibid., Lettre XV, 13. April 1658, S. 467).

2 Gazette de France, 12. August 1651, S. 828. 
Comte de Lionne in Berny empfangen: Begrüßt wurden die Gäste mit einem Konzert, auf das eine Jagdpartie folgte, an die sich die Aufführung einer Komödie anschloss. Daraufhin wurden die Gäste bei einem Bankett gestärkt, um nach einer Ballettaufführung und einem Feuerwerk selbst an einem Ball teilzunehmen, der mit einem Imbiss seinen Abschluss fand ${ }^{3}$.

Anlässe für die feierlichen Lustbarkeiten gaben auswärtige Besuche, Friedensschlüsse, Einweihungen und Grundsteinlegungen ebenso wie Geburtstage oder Hochzeiten in der königlichen Familie. Es war jedoch nicht üblich, von sich aus eine Einladung an den König auszusprechen. Vielmehr musste der Wunsch nach einem Fest von dem Monarchen selbst ausgehen. Für die Gäste bedeuteten die Feste eine Flucht aus der Eintönigkeit und Langeweile des Alltags ${ }^{4}$. Für den Gastgeber aber waren sie ein wichtiges Politikum: Zum einen steigerte bereits die Tatsache, dass der König von ihm empfangen zu werden wünschte, sein Ansehen in der Gesellschaft. Zum anderen bedeutete die offene Gunstbezeigung durch den Monarchen die Möglichkeit für eine weitere Protektion, und so verband sich die Festorganisation nicht selten mit der Hoffnung auf einen beruflichen und gesellschaftlichen Aufstieg. Unter Ludwig XIV. war René de Longueil nach einem zweifachen Bankett in seinem neu erbauten Schloss Maisons im April 1651 (dem ersten Empfang am 18. April folgte auf ausdrücklichen Wunsch des Königs zwei Tage später eine zweite Einladung) mit der Ernennung zum Marquis belohnt worden. Für das weit aufwendigere Fest, das der Comte de Lionne im Mai 1659 in seinem Schloss Berny gab, wurde dieser mit einem Staatsministerposten belohnt ${ }^{5}$. Ludwig hatte Fouquet bereits mehrere Wochen vor dem Fest vom 17. August 1661 anlässlich des Verkaufs seines Amtes als Generalstaatsanwalt die Nachfolge des Kanzlers Séguier in Aussicht gestellt. Der Finanzminister konnte daher nach der Bitte des Königs um eine Einladung nach Vaux-le-Vicomte auf einen Aufstieg hoffen.

\section{Vorherige Feste bei Fouquet}

Das Fest vom 17. August 1661 war nicht der erste königliche Empfang durch den Finanzminister. Ab dem Jahr 1656 finden sich vermehrt Hinweise auf und Beschreibungen von festlichen Empfängen und Veranstaltungen in den Häusern von Fouquet in den Gazetten ${ }^{6}$. Vor der Fertigstellung des Rohbaus von Vaux-le-Vicomte luden die Fouquets ihre illustren Gäste zu Festen nach

3 Ibid., 24. Mai 1659, S. 491f.; Muze historique, Bd. III, Lettre XX, 24. Mai 1659, S. $57 f$.

4 Alewyn, Feste, S.245.

${ }^{5}$ Muze historique, Bd.III, Lettre XXV, 28. Juni 1659, S.71: »Monsieur le Comte de Lionne a si bien servy la Couronne, que par un juste Rézultat, on l'a fait Ministre d'Estat «.

${ }^{6}$ Die »Muze historique «, in der die Festbeschreibungen der Pariser Gesellschaft allgemein einen großen Raum einnehmen, und die »Gazette de France « berichteten von den Festen in den Häusern des surintendant des Finances. 
Saint-Mandé. Ab dem Jahr 1659 wurden die herrschaftlichen Empfänge nach Vaux-le-Vicomte verlegt. Im Juni dieses Jahres besichtigte Mazarin das neue Schloss, in dem nach dem Einzug Le Bruns im September 1658 mit der Innendekoration begonnen worden war. Kurz darauf, am 17. Juli 1659, wurde erstmals auch der König in Begleitung seiner Mutter »et toute leur nombreuze suite $\ll^{7} \mathrm{zu}$ einem Diner im Schloss empfangen ${ }^{8}$. Den Charakter dieses Besuches beschreibt Fouquet selbst in einem Brief an Mazarin vom 19. Juli 1659:

Le roi, la reine, Monsieur, voulurent venir à Vaux voir mes fontaines jeudi dernier sur ce que Leurs Majestés avoient ouï dire que V.E. ne les avoit pas trouvées désagréables. Je crus être obligé de m'y trouver et de leur faire préparer la collation sans autre cérémonie. La journée se trouva belle et Leurs Majestés parurent fort satisfaites du lieu et me firent l'honneur de me traiter avec beaucoup de bonté et de civilité9.

Kurz nach seiner Vermählung - noch vor seinem feierlichen Einzug in Paris kam Ludwig am 19. Juli des folgenden Jahres mit seiner jungen Gemahlin Maria Theresia ein weiteres Mal zu Fouquet, der ihm nun einen weitaus glänzenderen Empfang bescherte und es nicht, wie bei dem letzten Besuch, bei einem Imbiss belie $\beta^{10}$. Le Brun erhielt die Anweisung, seine Arbeit als Innendekorateur einzustellen und als Maschinist und Dekorateur für die Zerstreuung der Gäste zu sorgen. Mit einer innovativen pyrotechnischen Inszenierung versetzte er die Gäste in Staunen:

Sur la fin d'un magnifique souper qui dura bien avant dans la nuit, on vit paraître tout à coup en l'air la représentation d'une lune qui jetoit une lumière si éclatante à mesure qu'elle s'élevoit sur l'horizon, que le roi et toute la cour demeurèrent longtemps surpris d'un spectacle si nouveau, et chacun en donna des applaudissements à M. Le Brun ${ }^{11}$.

Am 12. Juli 1661 wurde, auf ausdrücklichen Wunsch Ludwigs XIV., erneut königlicher Besuch in Vaux-le-Vicomte empfangen: Die englische Königinwitwe Henrietta Maria erschien in Begleitung ihrer Tochter sowie ihres Schwieger-

\footnotetext{
7 Muze historique, Bd. III, Lettre XXIX, 26. Juli 1659, S. 82.

${ }^{8}$ In der »Gazette de France « (24. Juli 1659, S.714) heißt es zu dem Fest: »Fouquet [...], qui les régala \& toute leur Suite, d'une Collation de toutes sortes de viandes, fruits \& confitures les plus rares, \& en telle abondance, qu'il ne se peut rien voir de si magnifique, non plus que si bien ordonné, ni de si agréable, par l'éclat \& la beauté de ce superbe Lieu, qu'on scait être des plus délicieux \& des plus charmans«.

9 Colbert, Lettres, Bd. I, S. 504.

${ }^{10}$ Muze historique, Bd. III, Lettre XXIX, 24. Juli 1660, S.231: »Dans sa belle Maizon de Vaux, où, par ses soins et ses travaux et ses honorables dépences, paroissent cent magnificences, soit pour la structure, ou les eaux, pour les dorures, ou tableaux, ou pour les jardins délectables, qui ne sont pas moins qu'admirables. Ce fut, donc, en ce lieu pompeux, que bien décrire je ne peux, dautant qu'il passe ma portée, que ladite Cour fut traitée: mais (outre le zèle et l'ardeur) ce fut avec tant d'abondance, et, mesme, en si belle ordonnance, que les Banquets d'Assuérus, prédécesseurs du Grand Cyrus, soit pour les pâtures exquizes, soit pour les rares friandizes, les brûvages, les fruits, les fleurs, conserves, de toutes couleurs, fritures et paticeries, n'êtoient que de gargoteries, en comparaizon du Banquet que fit, alors, Monsieur Fouquet«.

11 Guillet de Saint-George, Mémoires, Bd. I, S. $20 \mathrm{f}$.
} 
sohnes, des Herzogs von Orléans, dem Bruder Ludwigs ${ }^{12}$. Im Vergleich zu den bisherigen Festen im Hause Fouquet wurde an diesem Tag mit einem weit größeren Aufwand für die Zerstreuung der Gäste gesorgt ${ }^{13}$. Der Abend begann mit einem Festmahl, anschließend wurden die Gäste unter Musikbegleitung in den Garten geführt. Die Aufführung der »École des maris« von Molière im salon des Muses bildete den Höhepunkt des Festes ${ }^{14}$. Dieses Stück, welches erst drei Wochen zuvor in Paris uraufgeführt worden war, fand einen solchen Zuspruch, dass es bereits am Morgen des nächsten Tages in Fontainebleau vor dem König und am selben Abend erneut vor Mme Fouquet gespielt wurde.

Der König, der dem Fest in Vaux-le-Vicomte nicht hatte beiwohnen können, wünschte, nun auch die nahezu fertige Schlossanlage zu besichtigen ${ }^{15}$.

\section{Das Fest vom 17. August 1661}

Der Wunsch des Königs, von seinem Finanzminister in Vaux-le-Vicomte empfangen zu werden, gab diesem die Chance, sich dem König als loyaler Diener zu beweisen. Zugleich gab ihm das Begehr des Monarchen Hoffnung auf eine Festigung seiner Stellung, die für ihn umso wichtiger war, da er sein Amt als Generalstaatsanwalt zur Befriedigung der privaten Geldwünsche Ludwigs verkauft hatte.

Nur wenige Wochen blieben Fouquet nach dem Besuch aus England für die Vorbereitung eines Festes, das ihm die Möglichkeit gab, dem jungen Monarchen sein Schloss und sich auf eine neue Weise zu präsentieren. War bis zum März 1661 noch die Übermittlung der ikonographischen Botschaft von Vaux das Hauptanliegen des Finanzministers gewesen, so musste er nun versuchen, die hinfällige Symbolik der Deckenmalereien durch eine innovative Festgestaltung zu kompensieren.

Bereits kurz nach dem 12. Juli begannen die Vorbereitungen für den Empfang Ludwigs. Die Organisation des Abends stellte Fouquet unter die Auf-

12 Fouguet, Euvres, Bd.VI, S.137: »Le Roy m'ayant fait l'honneur de m'avertir, peu avant le voyage de Nantes, qu'il desiroit que la Reine d'Angleterre, Monsieur \& Madame y fussent receus «.

13 Die »Muze historique « berichtet ausführlich von diesem Fest. Der Ausgabe vom 17. Juli 1661 (Bd. III, Lettre XXVIII, S.377f.) sind ganze 73 Verszeilen gewidmet, wobei rund 50 Verse den Hausherrn und sein Schloss loben. Eine Ausgabe der »Muze historique« umfasste für gewöhnlich 200-250 Verse.

14 Ibid.: »Aprés qu'on eut de pluzieurs Tables desservy cent mets délectables tous confits en frainds apas, qu'icy je ne dénombre pas: Outre concerts et mélodie, il leur donna la Comédie; sçavoir l'Escole des Maris, charme (à prézent) de tout Paris, piéce nouvelle et fort prizée, que sieur Molier a compozée«.

15 Fouquet, CEuvres, Bd. VI, S. 137: »Depuis sa Majesté même, la Reine Mere, \& toute la Cour ayant fait dessein d'y venir«. 
sicht François Vatels ${ }^{16}$ und Charles Le Bruns. Ihre Arbeit wurde von mehreren Künstlern unterstützt. Torelli, italienischer Dekorateur und Maschinist, übernahm die Bühnengestaltung ${ }^{17}$. Molière erhielt den Auftrag zu einem neuen Stück und verfasste mit »Les Fâcheux« erstmalig (und zum letzten Mal) eine Komödie im Auftrag Fouquets ${ }^{18}$. Ohne die Aufführungen der »École des maris« in Paris zu unterbrechen, gelang es Molière, das Bühnenstück in kürzester Zeit zu schreiben und einzustudieren. Nur drei Tage vor dem Fest reiste er mit seiner Truppe nach Vaux und begann mit den Proben zu den »Fâcheux « ${ }^{19}$. Pellisson verfasste den Prolog zu der neuen Komödie, Beauchamp komponierte die Musik für ein Ballett.

Quellenbelege für die baulichen Vorkehrungen des Festes enthalten die »copies de quittances délivrées par divers artistes à Le Brun pour le paiement

16 Vatel trat 1653 zunächst als écuyer de cuisine (Küchenknappe) in den Dienst Fouquets und wurde von diesem bald zum maître d'hôtel befördert. Damit vergrößerte sich sein Zuständigkeitsbereich erheblich. Er war von nun an für die Organisation des gesamten Fouquetschen Haushaltes zuständig. Er verhandelte mit den Lieferanten, bewachte die Arbeiten im Hause, stellte das Personal ein und fand zudem als Vertrauensmann für delikate Missionen Verwendung. Er gilt als der Organisator des Festes vom 17. August 1661 (vgl. Pitte, »Vatel«, in: DGS, S.1566f.).

17 BuURSTRÖM, Torelli, S. 180: »As a matter of fact Torelli was the only one of those who participated in arranging major court spectacles who was willing to undertake the task. Lully had declined it, probably because he was aware of the increasingly precasious nature of Fouquet's position«. Es handelt sich bei dieser Festgestaltung um den letzten Auftrag Torellis in Frankreich.

18 Seit 1656 hatte Fouquet von den Erfolgen des Autors des »Étourdi ou les Contretemps « in Lyon gehört, doch selbst nachdem ein erstes Stück der Molièreschen Truppe, »Les Précieuses ridicules«, auch in Paris aufgeführt worden war, zeigte er kein großes Interesse an den Arbeiten des jungen Molière. Mit einer Aufführung der »Précieuses« im Hause M. de Guenegards im Februar 1660 und rund drei Monate später bei M. d'Andilly hielten die »Précieuses« auch in die Salons Einzug. Den ersten Hinweis auf einen Besuch Molières bei Fouquet gibt es für den Herbst 1660. Das breite Interesse an der Schauspielkunst der Truppe, die mittlerweile sowohl vor dem königlichen Hof als auch im Hause Mazarins und anderer hoher Persönlichkeiten spielte, hatte auch Fouquet neugierig gemacht. Seine Versuche, Molière mitsamt seiner Truppe zu engagieren, zeigten trotz allgemeiner Nachfrage Erfolg, da die Höhe seines Angebotes alle anderen Gehaltsofferten übertraf: Fouquet zahlte das Doppelte der üblichen Gage. Die erste Aufführung im Hause Fouquets fand am 11. Oktober statt. Der Darbietung von »Sganarelle ou le Cocu imaginaire« folgte die Aufführung des »Étourdi«. Molière selbst spielte Sganarelle und präsentierte in dieser Rolle seine ganze Schauspielkunst, die ein Zeitgenosse mit den folgenden Worten beschreibt: "L'on n'a jamais vu tenir des discours aussi naïfs, ni paroître avec un visage si niais et l'on ne doit pas moins admirer l'acteur pour avoir fait cette pièce que pour la manière dont il la représente. Jamais personne ne sut si bien démonter son visage et l'on peut dire que dans cette pièce il en change plus de vingt fois « (Neufvillaine über die Szene XII der »Sganarelle«, zitiert nach: Molı̀̀re, Euvres complètes, S. 250). In der Folge kam es zu weiteren Aufführungen im Hause Fouquets. In Vaux spielte die Molièresche Truppe erstmalig auf dem Fest vom 12. Juli 1661.

19 Muze historique, Bd.III, Lettre XXXIII, 20. August 1661, S.392, Vers 125-132: »Sur ce Téatre, que je dis, qui paroissoit un Paradis, fut, avec grande mélodie, récitée une Comédie, que Molier, d'un esprit pointu, avoit compozée, in promptu, d'une maniére assez exquize, et sa Troupe, en trois jours, aprize«. 
de travaux exécutés à Vaux-le-Vicomte ${ }^{20}$. Am 12. August stellte der Maurermeister Jacques Prou eine Quittung für Arbeiten aus »faict pour Monseigneur le Surintendant en son chasteau de Vaulx pour les chassis à mettre les lumières qui doivent estre pozé dans les croisées au pourtour du chasteau et pour les planches que j'ay fourny pour les loges aux danseurs et commediens « ${ }^{21}$. Am 17. August 1661 quittierte der Bildhauer Nicolas Legendre ebenfalls den Erhalt seines Lohnes für Arbeiten »que nous avons faict, Monsieur Girardon et moy, pour la maison à Vaux-le-Vicomte le dixseptième aoust «22. Um das noch nicht vollendete Anwesen mit dem nötigen Mobiliar auszustatten, räumte Fouquet sein Schloss in Saint-Mandé sowie sein Pariser Hôtel d'Émery und ließ mit Möbeln, Tapisserien, feiner Wäsche und silbernem Tafelgeschirr beladene Lastwagen nach Vaux kommen:

Je fus obligé, pour les recevoir, d'y faire porter tous les meubles que j'avois, et d'en acheter encore. Toutes les années précédentes, on sait que Leurs Majestés ou Monsieur le Cardinal, des ambassadeurs, et d'autres personnes par son commandement, y étoient reçues; en sorte que je fus contraint, d'incommoder mes affaires pour avoir de la vaisselle et des meubles dix fois au delà de ce que j'en eusse eu sans ses ordres fréquents, et sans que je croyois devoir rendre au Roi le respect le plus grand que je pouvois, et que je faisois chose agréable à Sa Majesté de m'acquitter honorablement de toutes ces choses, quoiqu'elles me fussent onéreuses ${ }^{23}$.

Am frühen Abend des 17. August war alles in Vaux-le-Vicomte bereit, den aus Fontainebleau kommenden König und seinen Hof zu empfangen. Vier Quellen geben eine Beschreibung von dem großen Fest: Jean de La Fontaine berichtet in der in einem Brief an seinen Freund Maucroix überlieferten »Relation d'une fête donnée à Vaux« von dem Abend ${ }^{24}$. Eine lange Zeit als anonym eingestufte Beschreibung ist die mittlerweile André Félibien zugeschriebene »Relation des magnificences faites par Monsieur Foucquet à Vauxle-Vicomte lorsque le roy y alla, le 17 août 1661, et de la somptuosité de ce lieu $\aleph^{25}$. Auch in der »Muze historique « von Jean Loret sowie in der »Gazette de France $\ll$ finden sich Beschreibungen des Festes ${ }^{26}$.

\subsection{Der Festverlauf}

Am 17. August 1661 erreichte der aus Fontainebleau kommende Hof gegen 18 Uhr Vaux-le-Vicomte. Von Trommelklängen und Musketieren begleitet

${ }^{20}$ Archives de Vaux-le-Vicomte, abgedruckt in: CORDEY, Vaux-le-Vicomte, Dokument XI, S.232-238.

21 Ibid., S. 235.

22 Ibid., S. 236.

${ }^{23}$ Fouquet, Euvres, Bd. VI, S.137f.

${ }^{24}$ La Fontaine, Euvres complètes I, Bd. VIII, S. 342-352.

25 BN Collection Morel de Thoisy, Bd. CDII, fol. 714-736, abgedruckt in: Cordey, Vaux-leVicomte, S.191-194 und in: FéLIBIEN, Relations, Relation des magnificences, S.31-33.

26 Gazette de France, 18. August 1661, S. 798. 
führte der junge König Ludwig XIV. »ayant avec lui, dans sa Calèche, Monsieur, la Comtesse d'Armagnac, la Duchesse de Valentinois, \& la Comtesse de Guiche ${ }^{27}$ den Zug an. Ihnen folgten Madame, »qui alloit en litière dans le soupçon d'une grossesse $\ll^{28}$ und die Königinmutter in ihrer Kutsche mit ihren Hofdamen. Den Schluss des Trosses bildete der Hofstaat mit M. Le Prince, M. De Longueville, M. Le Duc, M. De Beaufort, M. De Guise ${ }^{29}$ und einer Vielzahl weiterer Damen und Herren: »Cent objets miraculeux, des Grands Princes, ces Cordons-bleus, tous gens choizis et d'importance, bref, la fleur de toute la France $\ll^{30}$. Erschöpft von der Fahrt in der sommerlichen Hitze, begab sich die Festgesellschaft zunächst in das Schloss. Hier erwartete die Besucher ein prunkvolles Interieur. Das gesamte Haus war ausgestattet mit teurem Brokat, Spiegeln, mit Gold und Silber besetzten Stoffen, mit chinesischer Seide bezogenen Fauteuils, persischen Teppichen, Silber- und Goldvasen, Porphyr- und Marmortischen mit vergoldeten Füßen, kristallenen Schalen und Kerzenständern, Goldschmiedekunst und Uhrwerken: »Les meubles sont splendides et somptueux dans les appartemens et Leurs Majestez s'y reposèrent jusqu'à ce que le soleil fut baissé «31.

Nachdem sich die Hitze des Tages gelegt hatte, folgte der Schloss- die Gartenbesichtigung. Bei der Vorbereitung des Spazierganges hatte Fouquet sorgfältig bedacht, den hohen Gästen trotz des in Terrassen angelegten Gartens jeden Winkel zugänglich zu machen: »Les calèches qu'il avoit fait faire passoient partout, et la reine mère fit toute la promenade en calèche « ${ }^{32}$. Als es dunkel geworden war, kehrte man in das Schloss zurück. Nun begannen die eigentlichen Festlichkeiten, die mit einem prächtigen $a m b i g u^{33}$ eröffnet wurden: »Non de jambons, ny d'aloyaux, mais d'infinité de viandes si délicates, si friandes, y compris mille fruits divers, les uns sucrez, les autres verds, que

27 Ibid.

${ }^{28}$ FÉLIBIEN, Relations, Relation des magnificences, S. 31. La Fontaine gibt in seinem Brief an Maucroix an, dass Maria Theresia »étoit demeurée à Fontainebleau pour une affaire fort importante: tu vois bien que j'entends parler de sa grossesse (LA Fontaine, Euvres complètes I, Bd.VIII, S.345). Aufgrund einer Fehlgeburt Annas von Österreich war es der jungen Königin untersagt worden, große Reisen und Anstrengungen auf sich zu nehmen. Am 1. November wurde der Dauphin Louis geboren.

${ }^{29}$ FÉLIBIEN, Relations, Relation des magnificences, S. 31.

30 Muze historique, Bd. III, Lettre XXXIII, 20. August 1661, S. 391, Vers 37-40.

31 Félibien, Relations, Relation des magnificences, S.31. Zur Ausstattung von Vaux-leVicomte siehe auch: BN ms. fr. 7620, fol.106-146, Inventaire de Vaux (1661), in Auszügen abgedruckt in: BonNaFFÉ, Foucquet, S.77-96.

32 FÉlibien, Relations, Relation des magnificences, S.32. Chatelain merkt durchaus zutreffend an, dass dieser Spaziergang und die durch ihn hervorgerufene Begeisterung Ludwigs für die zur Perfektion geführte und von den Italienern inspirierte Gartenarchitektur den König dazu verführte, die Pracht Vaux' zunächst auf Versailles und später auf Marly zu übertragen: »Qui saura jamais combien de millions a coûté à la France cette promenade de Louis XIV?«(Chatelain, Foucquet, S.473).

${ }_{33}$ Bei einem ambigu handelt es sich um eine Mahlzeit, bei der alle Gerichte zugleich aufgetragen werden. 
cela (choze très-certaine) passe toute croyance humaine « ${ }^{34}$. Fasane, Ortolane, Wachteln, Krustentiere, Ragouts und andere Köstlichkeiten wurden den Gästen serviert ${ }^{35}$. Ein Violinkonzert untermalte das Festmahl musikalisch. Alles war im Überfluss vorhanden, »les tables furent relevées plus de cinq ou six fois et il n'y eut personne qui n'en fust pleinement satisfait « ${ }^{36}$.

Gestärkt von dem exquisiten Mahl begab man sich erneut in den Garten, um der Uraufführung der »Fâcheux« von Molière beizuwohnen. Die Bühne befand sich am Ende des östlichen kleinen Kanals zwischen erster und zweiter Parterreebene vor der grille d'eau, dem Gitter aus Wasserstrahlen:

En cet endroit qui n'est pas le moins beau

De ceux qu'enferme un lieu si délectable,

Au pied de ces sapins et sous la grille d'eau,

Parmi la fraîcheur agréable

Des fontaines, des bois, de l'ombre, et des zéphyrs,

Furent préparés les plaisirs

Que l'on goûta cette soirée.

Des feuillages touffus la scène étoit parée,

Et de cent flambeaux éclairée :

Le Ciel en fut jaloux. Enfin figure-toi

Que lorsqu'on eut tiré les toiles,

Tout combattit à Vaux pour le plaisir du Roi :

La musique, les eaux, les lustres, les étoiles ${ }^{37}$.

34 Muze historique, Bd. III, Lettre XXXIII, 20. August 1661, S. 392, Vers 110-116.

35 Um die Gäste zu bewirten, fanden rund 6000 Teller, 432 Schalen und ein komplettes feuervergoldetes Silberservice Verwendung (vgl. PetrTFils, Fouquet, S. 356). Meyer (Fouquet, S. 14) schließt aus diesen Angaben auf eine Anzahl von rund 6000 Gästen. Dies ist eine utopische Zahl. Man kann vielmehr davon ausgehen, dass der königliche Hof aus Fontainebleau mit 350-400 Personen anreiste (dieser Annahme liegen folgende Schätzwerte zugrunde: das Gefolge des Königs umfasste im Jahre 1661 circa 140, jenes der Königinmutter 60 Personen; Monsieur und Madame reisten in Begleitung von je 40 Personen, das Gefolge der princes du sang und der princes étrangers umfasste circa 70 Personen; die Zahl der den Hof begleitenden Minister, Botschafter und wichtigsten Militärs kann auf 40 Personen geschätzt werden). Nimmt man an, dass in Vaux selbst bereits einige Gäste den Hof erwarteten, kann man davon ausgehen, dass bei dem Fest insgesamt circa 500 Personen anwesend waren.

${ }^{36}$ FéLibien, Relations, Relation des magnificences, S.32. Quellen aus dem 18. Jahrhundert zufolge riefen nicht nur die Speisen an diesem Abend die grenzenlose Bewunderung der Gäste hervor. Dézallier d'Argenville (Voyage pittoresque, S.210f.) berichtet in seiner »Voyage pittoresque des environs de Paris« »que du plafond de cette salle les tables descendaient magnifiquement servies et [...] qu'il s'élevait auparavant un brouillard qui en dérobait la vue aux convives«. GoudemeTz, Voyage, S. 190 fügt hinzu: »On remarque encore au plafond les écrous par le moyen desquels on faisait descendre pour la bouche du monarque le nectar des dieux et mille genres de friandises«. Hierzu Pérouse DE Montclos, Vaux-le-Vicomte, S.166: »En prenant compte le fait que le montage actuel du plafond est sommaire, on ne peut exclure que des tables volantes aient été un temps utilisées «.

37 La Fontaine, Euvres complètes I, Bd. VIII, S. 346. 
Die Dekorationen, entstanden in Zusammenarbeit Le Bruns und Torellis, »furent magnifiques $\ll^{38}$. Das natürliche Dekor der Parkanlage, sowohl die die Bühne umgebenden Bäume und Sträucher als auch die hinter dem Podium emporschießenden Wasserstrahlen und die dahinterliegende Allee, wurde bei der Bühnengestaltung einbezogen. Zusammen mit wenigen beweglichen Bühnenstücken bildeten sie das Bühnenbild ${ }^{39}$. Bei der Konzipierung seines Stückes hatte Molière bedacht, dass das Dunkel der Nacht überraschende Momente am besten zur Geltung bringen konnte. Da er sich vorgenommen hatte, den jungen König die Rolle eines Zauberers spielen zu lassen und der Aufführung damit einen märchenhaften Zug zu verleihen sowie den König als Ehrengast einzubeziehen, erschien Molière zunächst in Alltagskleidern und einem betrübten Gesicht auf der Bühne: »S'adressant au Roi avec le visage d'un homme surpris, [Molière] fit des excuses en désordre sur ce qu'il se trouvait là seul, et manquait de temps et d'acteurs pour donner à Sa Majesté le divertissement qu'elle semblait attendre, si quelque secours étranger ne lui arrivoit « ${ }^{40}$.

Molière bat den König, ihm zu Hilfe zu kommen; sogleich befahl dieser durch ein Zeichen seiner Hand, dem betrübten Künstler aus der Misere zu helfen. Das Signal des Königs schien magische Kräfte zu haben:

D'abord aux yeux de l'assemblée

Parut un rocher si bien fait

Qu'on le crut rocher en effet;

Mais, insensiblement se changeant en coquille,

Il en sortit une nymphe gentille

Qui ressembloit à la Béjart ${ }^{41}$,

Nymphe excellente dans son $\operatorname{art}^{42}$.

Mit Anmut begann die Nymphe, einen Prolog zu Ehren des jungen Herrschers zu rezitieren ${ }^{43}$ und befahl den sie umgebenden Statuen im Namen des Königs, aus dem Marmor, der diese umschloss, zu entweichen und sich mit all ihren Kräften an der Zerstreuung Seiner Majestät zu beteiligen. Alle Statuen, die gerade noch einen Teil der Bühnendekoration dargestellt hatten, begannen nun sich zu bewegen und in Faune und Bacchanten zu verwandeln. Auf

\footnotetext{
38 Ibid.

39 Bjurström verweist auf genaue Stellenangaben in dem Theaterstück selbst, in denen auf Teile des Parks hingewiesen wird. So sagt Lysandre zu Beginn von Akt 1, Szene 3: »Sous ces arbres, de loin, mes yeux l'ont reconnu«. Er verweist zugleich auf einen in der Nationalbibliothek in Wien aufbewahrten Bühnenentwurf, den er für einen möglichen Entwurf für die Aufführung von »Les Fâcheux « in Vaux-le-Vicomte hält (vgl. BJUrströM, Torelli, S.180-183).

40 Avertissement des »Fâcheux«, in: Molıère, Euvres complètes, S. 408.

${ }^{41}$ Madeleine Béjart (1618-1672) zählte mit ihren Geschwistern Joseph und Geneviève sowie ihrem jungen Liebhaber Jean-Baptiste Poquelin (Molière) zu den Gründern des Illustren Theaters und erwarb sich als herausragende Schauspielerin Ruhm und Ansehen. ${ }^{42}$ La Fontaine, Euvres complètes I, Bd. VIII, S. 347.

${ }^{43}$ Dieser Prolog aus der Feder Pellissons ist bis heute im Gesamtwerk Molières den »Fâcheux« vorangestellt (MoLIÈre, Euvres complètes, S. 409).
} 
einen weiteren Befehl der anmutigen Nymphe befreiten sich alsbald auch die Bäume aus ihrer Erstarrung und nahmen die Gestalt von Dryaden und Satyrn an. Begleitet von den Klängen mehrerer Violinen und Oboen ging die Verwandlungsszene in das Entrée des Balletts über, welches sich in der Folge im Wechsel mit komödiantischen Szenen der »Fâcheux« zu einer neuen Gattung entwickelte, die von Molière eigens aus Anlass des Festes entwickelt worden war: die Ballettkomödie. Beauchamp war mit der musikalischen Leitung betraut worden und hatte sowohl die Musikstücke komponiert als auch die Balletteinlagen choreographiert ${ }^{44}$.

Der Aufführung folgte unmittelbar ein nächstes Spektakel: ein von Trommelschlägen rhythmisiertes gigantisches Feuerwerk. Aus dem Amphitheater, welches die Grotte überragte, begannen Lichtstrahlen in den Himmel zu schießen:

Figure-toi qu'en même temps

On vit partir mille fusées,

Qui par des routes embrasées

Se firent toutes dans les airs

Un chemin tout rempli d'éclairs,

Chassant la nuit, brisant ses voiles.

As-tu vu tomber les étoiles?

[...] Parmi ce spectacle si rare,

Figure-toi le tintamarre,

Le fracas, et les sifflements,

Qu'on entendoit à tous moments ${ }^{45}$.

Das von Torelli inszenierte Feuerwerk umhüllte die Gäste mit Zischen und Krachen. Die Lichtraketen nahmen in der Luft die verschiedensten Formen an: »Plus de quatre cens fleurs de Lys, dont des bords etoient embellis [...] outre seize grandes Figures qui n'étoient, pourtant, que Peintures de mesme compozition mais faites en perfection « 46 . Zugleich näherte sich auf dem großen Kanal die kunstvoll gestaltete Attrappe eines Wales, der ebenso unzählige Rauchraketen und Feuerwerkskörper entsprangen »de sorte qu'on s'imaginoit que le feu et l'eau, s'étant unis n'étoient qu'une seule et même chose. [...] Les Fusées, aprez avoir serpenté longtemps sur l'eau, s'élançant d'elles-mêmes en produisoit d'autres qui faisoient le mesme effet des premières $\ll^{47}$. Das gesamte Schauspiel wurde von Trompeten- und Trommelklängen begleitet, die den Eindruck verstärkten, man befände sich mitten in einer rasenden Schlacht. Félibien beschreibt die eindrucksvolle Darbietung wie folgt: »Je vous avoue que mon âme pacifique sentoit enfler son courage et

${ }^{44}$ Eine Courante geht bereits bei der Premiere des Ballett-Teils auf Lully zurück. Bei den späteren Aufführungen des Stückes in Versailles übernahm dieser die gesamte musikalische Ausgestaltung des Stückes und wurde in der Folge abgelöst von Charpentier.

45 La Fontaine, Euvres complètes I, Bd. VIII, S. 350.

46 Muze historique, Bd. III, Lettre XXXIII, 20. August 1661, S.392, Vers 213-222.

47 Félibien, Relations, Relation des magnificences, S.33. 
que je serois devenu guerrier, si l'occasion en eüt esté aussi véritable qu'elle étoit bien representée « 48 .

Nachdem der taghell gewordene Himmel wieder seine nächtliche Färbung angenommen hatte und die Stille der Nacht zurückgekehrt war, wurde die Festgesellschaft noch von einem finalen Feuerwerk überrascht. Aus dem Kuppelturm des Schlosses schossen Schwärmer und Schlangen in den Himmel und bildeten einen Bogengang, unter dem die Gäste hindurchschritten: »On crut que tous les astres, grands et petits, étoient descendus en terre ${ }^{49}$. Im Schloss erwartete den König und seinen Hof eine letzte Überraschung: $\mathrm{Zu}$ Violinmusik wurde erneut ein Imbiss gereicht »de toutes sortes de fruits les plus beaux et les plus rares [...] et toute la cour trouva que ce refraîchissement [...] étoit fort nécessaire ${ }^{50}$. Daraufhin bestiegen die Gäste die wartenden Kutschen und verließen, von Trommelschlägen begleitet, das festliche Vaux in Richtung Fontainebleau.

\subsection{Das Fest vom 17. August 1661: ein Fazit}

Das Fest vom 17. August 1661 übertraf die bisherigen königlichen Empfänge in Vaux-le-Vicomte und präsentierte das Schloss dem König erstmals als einen Ort vielfältiger Zerstreuungen. Die Entscheidung, einen Großteil des Festes im Garten stattfinden zu lassen, kam nicht von ungefähr. Zum einen war hier die hinfällig gewordene Ikonographie von Vaux am wenigsten präsent. Darüber hinaus konnte nur im Garten die große Gästeschar untergebracht werden, die in dem eher kleinen Schloss keinen Raum gefunden hätte. Zudem war der Garten der Teil der Anlage, der bereits in der ursprünglichen Konzeption von Vaux für das königliche Divertissement vorgesehen gewesen war. Mit den hier stattfindenden Aktionen wollte der Finanzminister den Monarchen für die Schlossanlage begeistern und seinen Bau rechtfertigen.

Fouquet war überzeugt, dass der junge König auch nach der Übernahme der Regierungsgeschäfte seine Begeisterung für alle Arten von Zerstreuungen nicht verloren hatte:

Il [Fouquet] se moqua d'eux [ses amis], les assurant que ces premières velléités de gouverner ne seraient pas longtemps dans l'esprit d'un jeune roi, entraîné par ses passions; et qu'il n'y avait guère d'apparence qu'il pût se soutenir huit heures par jour dans des occupations désagréables, lui que les plaisirs entouraient, et appelaient de tous côtés ${ }^{51}$.

\footnotetext{
48 Ibid.

49 La Fontaine, Euvres complètes I, Bd. VIII, S.351. Dieses letzte Feuerwerk forderte, vom Hofstaat unbemerkt, animalische Opfer: Zwei Zugpferde der Karosse der Königinmutter, die bereits zur Abfahrt bereitstanden, gingen durch und verletzten sich dabei so schwer, dass sie ihr Leben verloren (ibid.).

${ }^{50}$ FÉLIBIEN, Relations, Relation des magnificences, S.33.

51 Cholsy, Mémoires (2005), S. 125.
} 
Am Abend des 17. August wurden Ludwig XIV. daher in Vaux alle wichtigen Festelemente dargeboten, die ihn als Mittelpunkt der Anlage und des Festes inszenierten. Das eigens für diesen Anlass verfasste Stück Molières schrieb dem König übernatürliche Kräfte zu, indem die Aufforderung Molières den Monarchen die Verwandlung der Statuen und Steine in Nymphen, Faune und Bacchanten bewirken ließ. Überdies wurde das Lustspiel mit dem vom König bevorzugten Tanz zu einer Ballettkomödie verknüpft ${ }^{52}$. Die Erfindung dieser neuen Gattung war aus der Not geboren. Da man nur über eine kleine Gruppe von Tänzern und Schauspielern verfügte, hatte Molière die Idee, die Entrées des Balletts vor die einzelnen Akte der Komödie zu setzen, »afin que ces intervalles donnassent temps aux mêmes baladins de revenir sous d'autres habits « ${ }^{53}$. Akt eins und drei der Komödie wurden von je zwei Entrées abgelöst, auf den zweiten Akt folgten gar vier Balletteinlagen: »On avoit accommodé le ballet à la comédie, autant qu'il étoit possible, et tous les danseurs y représentoient des fâcheux de plusieurs manières « ${ }^{54}$. Das Theaterstück selbst setzte eine Reihe von aufdringlichen Personen in Szene, die ein junges Paar daran hinderten, ihre geheimen Verabredungen einzuhalten: »un marquis prétentieux, un cavalier galant, un amateur passionné de musique, un gentilhomme chatouilleux sur l'honneur, un joueur, deux précieuses bavardes, un cuistre pédant, un inventeur dans le rêve de ses imaginations, un bretteur professionnel $\ll^{55}$. Es ist zu vermuten, dass das Thema nicht rein zufällig gewählt wurde. Es spiegelte vielmehr die Situation wider, in der sich der Finanzminister sah, der von den Intrigen des Hofes daran gehindert wurde, zu einer ungetrübten Beziehung mit dem jungen Monarchen zu finden. Der Prolog hatte den König zu einem Akteur der Handlung werden lassen; dies sollte ihm zeigen, dass er auch im Alltag zu einem Handelnden werden und politische Störenfriede beseitigen konnte. Der imaginären Geschichte einer gestörten Zweisamkeit fehlte es nicht an einem subtilen Aktualitätsbezug.

Ähnlich verhielt es sich mit dem anschließenden Feuerwerk. Dieses machte nicht nur die Nacht zum Tag, sondern tat es überdies mit einer auf den König bezogenen Symbolik. Am Himmel zeigten sich Lilien, Namen sowie Sterne

52 Ein Hinweis auf einen Vorläufer der Ballettkomödie findet sich in der Muze historique, Bd.I, Lettre XV, 18. April 1654, S.485. Hier wird von einem Ballett berichtet, das der König tanzte: »Mardy, pour la premiére fois, Louis, la merveille des Rois, la gloire et l'honneur de la France, dansa son balet d'importance avec un si haut apareil qu'il n'en fut jamais de pareil. Le sujet êtoit, ce me semble, Comédie et balet ensemble, dont est auteur le sieur Bouty, de Rome expréssement party, pour, dans une splendeur extréme, charmer la Cour de ce beau poëme. Qui fut avec grand agrément récité muzicalement. Ladite piéce est apellée Nopces de Thétis et Pélée«. Ob sich bei dieser Aufführung Komödie und Ballett in gleicher Form zusammenfügten wie sieben Jahre später in Vaux-le-Vicomte, ist nicht überliefert. In der Literatur gilt »Les Fâcheux « allgemein als erstes Stück der neuen Gattung (Molière, Euvres complètes, S. 403).

53 Ibid.

${ }^{54}$ La Fontaine, Euvres complètes I, Bd. VIII, S. 349.

55 Notice sur »Les Fâcheux«, in: Molière, CEuvres complètes, S. $401 \mathrm{f}$. 
und andere Figuren, die wahrscheinlich ebenfalls in einem engen Bezug zum Monarchen standen ${ }^{56}$ : »De cet amphithéâtre sortit une quantité innombrable de fusées qu'on perdoit de vue et qui sembloient vouloir porter le feu dans la voûte des cieux, dont quelques-unes retombant faisoient mil figures, formoient des fleurs de lis, marquoient des noms et représentoient des étoiles «57.

Für die öffentliche Wirksamkeit war das Feuerwerk zunächst der wichtigste Teil des Festabends, da es eine Wirkung über den Kreis der Gäste hinaus erzielte $^{58}$. Nachträglich war auch die literarische Inszenierung des Festes ein wesentliches Mittel, eine breite Öffentlichkeit $\mathrm{zu}$ erreichen und die Großartigkeit des königlichen Empfanges durch Fouquet publik zu machen. Im Gegensatz zum Feuerwerk konnte die literarische Rezeption des Festes jedoch weit mehr erreichen: Sie vermochte zudem, die Wirkung des Abends auf die hohen Gäste, allen voran den König, vorwegzunehmen und damit die öffentliche Meinung zu beeinflussen. Jean de La Fontaine und André Félibien waren bereits vor dem Fest an der literarischen Inszenierung von Vaux-leVicomte beteiligt gewesen. Auch Jean Loret hatte schon vor 1661 die literarische Großinszenierung von Vaux ergänzt. Bereits seit 1656 fanden sich in seiner Salongazette, der »Muze historique«, ausführliche Berichte von festlichen Empfängen im Hause des Finanzministers. Mit einem Verweis auf die »Clélie« von Madeleine de Scudéry in der Ausgabe vom 17. Juli 1661 reiht er sich sogar in den Kanon der anderen Texte zu Vaux ein. Von Fouquet erhielt Loret eine regelmäßige Pensionszahlung, die ihn dem Minister verpflichtete. Die »Gazette de France« berichtete seit 1659 von Festlichkeiten bei den Fouquets, jedoch in einer äußerst knappen Form. Hinweise auf eine Förderung des Blattes durch den Finanzminister gibt es nicht. Es könnte sich bei der »Gazette« demzufolge um eine Quelle handeln, die nicht auf Initiative Fouquets hin und mit der Absicht, die Rezeption des Festes im Sinne des Finanz-

56 Obwohl von den Festbeschreibern nicht vermerkt, kann man davon ausgehen, dass neben Sternendarstellungen auch die Sonne pyrotechnisch nachgebildet wurde. MaGNE (Plaisirs, S.217) weist darauf hin, dass bereits 1649 anlässlich des elften Geburtstages des Königs am 5. September bei einem Feuerwerk die Sonne den Beginn seiner Herrschaft versinnbildlicht hatte. Magne spricht auf Seite 216 davon, dass sich allgemein mit der Geburt Ludwigs XIV. die Art der Feuerwerksdarbietungen verändert hatte. Gab es unter seinem Vater noch eine völlige Freiheit in der pyrotechnischen Gestaltung, so entwickelte sich unter Ludwig XIV. die allégorie pyrique, bei der man sich antiker Elemente ebenso bediente wie zeitgenössischer Wappen und Embleme.

57 FÉlibien, Relations, Relation des magnificences, S.33. In der »Muze historique (Bd. III, Lettre XXXIII, 20. August 1661, S.392, Vers 209-222) lautet es: »Pendant que ce grand Feu dura, que toute la Cour admira, je criay trente fois, miracle, ayant devant moy, pour spectacle, plus de quatre cens fleurs de Lys, dont des bords etoient embellis avec ordre et compas formées, et qui paroissans enflamées, sans consumer aucunement, excitoient du ravissement, outre seize grandes Figures, qui n'étoient, pourtant, que Peintures de mesme compozition mais faites en perfection «.

58 Magne, Plaisirs, S. 213: »Il constitue, avec les cortèges, un moyen [...] de se mêler, sans familiarité, au peuple, de donner à celui-ci part du plaisir, de tâter l'opinion. Plus loin, [...] l'Ancien Régime [...] l'utilisera à sa propagande politique«. 
ministers zu beeinflussen, verfasst wurde. Hierfür spricht auch die Kürze der Festbeschreibung, die nur wenige Zeilen einnimmt ${ }^{59}$.

Alle vier Beschreibungen beginnen mit einer ausführlichen Aufzählung der in Vaux empfangenen Gäste. Eine solch detaillierte Beschreibung der Festbesucher war durchaus nicht gewöhnlich ${ }^{60}$. Sie hob die politische Bedeutung des Festes in besonderem Maße hervor. Die tatsächliche Anzahl der Gäste lässt sich trotz der Ausführlichkeit der Quellen nur schätzen. Man muss davon ausgehen, dass rund fünfhundert Gäste bei dem Fest zugegen waren ${ }^{61}$. Die Zahl der an der Gestaltung des Abends beteiligten Personen war weit höher.

Die von Fouquet mit einer Festbeschreibung beauftragten Literaten La Fontaine, Félibien und Loret legen einen Schwerpunkt ihrer Texte auf die Aktionen, die im Garten stattfanden: Promenade, Theateraufführung und Feuerwerk. Das Schloss selbst wie auch die in ihm stattfindenden Teile des Festes finden wenig Beachtung, so dass der Empfang auch literarisch zu einem Gartenfest gerät.

Seiner »Relation« zufolge war Félibien beim Fest selbst anwesend ${ }^{62}$. Wie bereits im Falle der beiden zuvor verfassten Schriften über Vaux handelt es sich auch bei der Festbeschreibung um einen Brief an einen ungenannten Adressaten. Die »Relation« über den 17. August stellt demnach eine Ergänzung der vorhergehenden Arbeiten zu Vaux dar. Félibien liefert eine Festbeschreibung, die den König in den Mittelpunkt stellt und das Itinerar des Monarchen an diesem Abend rekonstruieren lässt. Er setzt Ludwig und seine Reaktionen auf das Fest in Szene. Zugleich nutzt Félibien seine Beschreibung - vor allem die des Gartenrundgangs - dazu, die Besonderheiten der Anlage zu schildern. Er rühmt den Wasserreichtum des Gartens und fügt seiner Darstellung einen Absatz bei, der die Abhebung der Anlage von italienischen Vorbildern unterstreicht ${ }^{63}$. Im Gegensatz $\mathrm{zu}$ den vorhergehenden Briefen steht in dieser »Relation« nicht die Beschreibung einzelner ikonographischer

${ }^{59}$ Gazette de France, 18. August 1661, S.798. Die Kürze des Textes könnte jedoch auch seiner unmittelbaren Entstehung nach dem 17. August geschuldet sein. Die Beschreibung erschien nur einen Tag nach dem Fest selbst. Von allen Festbeschreibungen liefert sie die ausführlichste Gästeliste.

${ }^{60}$ In der Beschreibung des Festes, das der Comte de Lionne am 18. Mai 1659 in Berny gab, führt Loret beispielsweise neben dem König und seiner Mutter lediglich »pluzieurs Belles de la Cour « und Mazarin an, obwohl insgesamt "plus de vingt-et-deux-mille « Menschen an dem Fest beteiligt waren - und auch die Gästeschar dementsprechend groß gewesen sein muss (Muze historique, Bd. III, Lettre XX, 24. Mai 1659, S.57).

${ }^{61} \mathrm{Vgl}$. Anm. 35 .

$62 »$ Je vous ay promis une relation de se qui passeroit à Vaux. Je serai bien heureux si ma mémoire peut fournir à tant de diverses choses que j'y ay veües. [...] Et pour moy, j'allay coucher à Melun ravi de tant de belles choses« (FéLIBIEN, Relations, Relation des magnificences, S.31-33).

${ }^{63}$ Ibid., S.32: »C'est icy [quarré d'eau] où il faut que Tivoli et Frascati et tout ce que l'Italie se vante de posséder de beau, de magnifique et de surprenant avouë qu'elle n'a rien de comparable à Vaux«. 
Attraktionen im Vordergrund, sondern die Schilderung der gesamten Anlage, so dass Vaux-le-Vicomte als Raum erfahrbar wird.

Auch La Fontaines Beschreibung des Festes weist Parallelen zu seinem ersten Text, dem »Songe de Vaux« auf. In einer Mischung aus Poesie und Prosa lässt er Vaux erneut als einen von Göttern und Fabelwesen belebten Ort entstehen. Doch ist auch in seinem Fall die Beschreibung nicht mehr auf die Person Fouquets, sondern auf den jungen Monarchen bezogen: »Je remarquai une chose à quoi peut-être on ne prit pas garde: c'est que les nymphes de Vaux eurent toujours les yeux sur le Roi; sa bonne mine les ravit toutes, s'il est permis d'user de ce mot en parlant d'un si grand prince « ${ }^{64}$.

Den Raum, den die Festbeschreibung in der »Muze historique « vom 20. August 1661 einnimmt, sprengt den gewöhnlichen Rahmen: Eine ganze Ausgabe hat Jean Loret dem Ereignis gewidmet; die normalerweise bis 250 Verse lange »Muze « umfasst an diesem Tag 298 Verse: »Aujourd'huy mes soins et travaux n'iront qu'à discourir de Vaux, Maizon, Rézidence, ou Retraite, qui n'est pas encore parfaite ${ }^{65}$. Seine Beschreibung des Festes erschien am 20. August 1661, nur einige Tage nach dem Ereignis. Sein Blatt, das eine breite Publikumswirkung hatte, erreichte die Öffentlichkeit also mit wenig Verzögerung und eignete sich somit hervorragend als Propagandamittel. Es kann daher kaum verwundern, dass Loret einen Schwerpunkt seiner Festbeschreibung auf die Beziehung zwischen Fouquet und dem König sowie die Lobpreisung der grenzenlosen Loyalität des Finanzministers setzte:

C'est ainsi que cét Homme sage,

Que cét Illustre Personnage,

Capable du plus haut Employ,

Fetoya son Maitre et son Roy,

N'épargnant ny soin, ny dépence,

Pour montrer sa magnificence;

Et j'ay sceu de quelques amis,

Que si le bref temps eut permis

D'achever maint sublime Ouvrage,

Il en eut bien fait davantage ${ }^{66}$.

Vor dem 17. August hatte Fouquet sein neues Schloss nicht nur literarisch, sondern auch graphisch inszenieren lassen. Es ist anzunehmen, dass - neben den Aufträgen an die Poeten von Vaux - auch die visuelle Rezeption des Festes in Auftrag gegeben worden war. Kein anderer als Silvestre, der zuvor be-

${ }^{64}$ La Fontaine, CEuvres complètes I, Bd. VIII, S. 345. Auf Seite 348 heißt es weiter: »La Béjart commande aux divinités [...] de contribuer de tout leur pouvoir au divertissement de Sa Majesté«.

65 Muze historique, Bd.III, Lettre XXXIII, 20. August 1661, S.391, Vers 17-20. Mir ist keine anderes Ereignis bekannt, dem Loret in seiner Salongazette eine ganze Ausgabe widmet. Selbst das mit dem Abend des 17. August 1661 vergleichbare vom Comte de Lionne in Berny am 18. Mai 1659 gegebene Fest umfasst nur einen (wenngleich großen!) Teil der Ausgabe vom 24. Mai 1659.

${ }^{66}$ Muze historique, Bd. III, Lettre XXXIII, 20. August 1661, S.393, Vers 243-252. 
reits die Stiche von Vaux angefertigt hatte, konnte dafür in Frage gekommen sein. Dem baldigen Sturz des Finanzministers mag es geschuldet sein, dass keine Stiche des Festes mehr entstanden sind.

Das Gartenfest vom 17. August 1661 stellte einen letzten Kraftakt des kranken Fouquet dar, seine Fähigkeiten in den Dienst des Königs zu stellen und zugleich mit der Festgestaltung die Diskrepanz zwischen der Symbolik der Schlossikonographie und der veränderten politischen Lage im Königreich aufzufangen. Indem er alle Festelemente an einem Abend zusammenführte und den Großteil der Veranstaltung in den Garten verlegte, wo Nymphen und Götter sowohl in den Statuen präsent waren, als auch in dem Prolog der Komödie aktiv wurden, der Wasserreichtum für eine anregende Atmosphäre sorgte und das Feuerwerk die Nacht zum Tag werden ließ, schuf Fouquet einen möglichst großen geistigen Abstand zwischen dem politischen Alltag und dem festlichen Abend in seinem Schloss. Für den Finanzminister war das Fest ein Ausdruck der Hoffnung, seinem politischen Schicksal noch eine Wende geben zu können.

Die Nacht des 17. August 1661 war nicht das erste Fest, auf dem dem jungen König Zerstreuungen in Form von Theateraufführungen, Balletteinlagen, Musik und Festmahlen geboten wurden. Dennoch setzte das Fest von Vaux den Markstein für eine neue Epoche der Festkultur, da hier zum ersten Mal alle traditionellen Bestandteile des höfischen Festes mit der Architektur des Gartens und des Schlosses zu einer Einheit verbunden und zudem in einer nie dagewesenen Perfektion vorgestellt wurden, die sich Ludwig zum Vorbild nehmen und die ihm als Anstoß für die drei großen Feste seiner ersten Regierungsphase dienen sollte. Das Fest von Vaux-le-Vicomte war damit der Ausgangspunkt der großartigen Versailler Feste, die im Jahre 1664 mit den »Plaisirs de l'île enchantée« ihren Anfang nehmen sollten ${ }^{67}$.

Dem Finanzminister allerdings brachte der glänzende Empfang des königlichen Hofes in Vaux-le-Vicomte keine zweite Chance. Er wurde nur wenige Tage nach der großen Festnacht am 5. September 1661 von königlichen Musketieren in Nantes verhaftet. Der Tag der Verhaftung stellte in doppelter Hinsicht ein bedeutendes Datum im Leben Ludwigs XIV. dar. Es war nicht nur der 23. Geburtstag des Monarchen. Mit der Verhaftung Nicolas Fouquets zog der König zugleich einen Schlussstrich unter die finanziellen Wirren seiner Jugendjahre und beendete eine Zeit der königlichen Schwäche. Der Sturz Fouquets wurde damit zu einem Symbol des politischen Umbruchs. Das pompöse Fest des Ministers gab dem König ein Mittel in die Hand, die bereits im Mai beschlossene Verhaftung zu legitimieren. Das Ende der Kulturpatronage Nicolas Fouquets bildete den Beginn der kulturellen Blüte Frankreichs unter Ludwig XIV.

67 Es folgten $1668 »$ Le Grand Divertissement royal de Versailles« und 1674 »Les Divertissements de Versailles «, beide beschrieben in André Félibiens »Relation de la fête de Versailles« (Paris 1994). 